Pacific Journal of Mathematic 


\section{ON TOEPLITZ MATRICES, A.BSOLUTE CONTINUITY, AND UNITARY EQUIVALENCE}

\section{R. Putnam}

1. Preliminaries. For $n=0, \pm 1, \pm 2, \cdots$, let $\left\{c_{n}\right\}$ be real numbers satisfying

$$
c_{0}=0, c_{-n}=c_{n} \text { and } \sum_{1}^{\infty} c_{n}^{2}<\infty \text {, }
$$

and consider the associated real-valued, even function $f(\theta)$ of period 2 and of class $L^{2}[0, \pi]$ defined by

$$
f(\theta) \sim \sum_{-\infty}^{\infty} c_{n} e^{i n \theta}=2 \sum_{1}^{\infty} c_{n} \cos n \theta .
$$

(Throughout this paper it will be assumed for the sake of convenience that $c_{0}=0$. If $c_{0} \neq 0, T$ (see below) is modified merely by the addition of a multiple of the unit matrix.) Let $A=\left(a_{j}\right)$, where $a_{i j}=c_{i-j}\left(=c_{j-i}\right)$ or $a_{i j}=0$ according as $i<j$ or $i \geq j(i, j=1,2, \cdots)$, and define the Toeplitz matrix $T$ and the Hankel matrices $H$ and $K$ by

$$
T=\left(c_{i-j}\right)=A+A^{*}, H=\left(c_{i+j-1}\right) \text { and } K=\left(c_{i+j}\right) \text {. }
$$

The matrices $T, H$ and $K$ are real and Hermitian (symmetric).

Let $J$ denote the matrix belonging to the quadratic form $2 \sum_{1}^{\infty} x_{n} x_{n+1}$. The differential of its spectral matrix is given by $d \rho_{i j}(\theta)=2 \pi^{-1} \sin i \theta$ $\sin j \theta d \theta$ (cf. Hilbert [5], p. 155, Hellinger [8], pp. 148 ff.). A direct calculation (cf. [11], Appendix 2) shows that

$$
T=F+K,
$$

where $T$ and $K$ are defined by (3), and $F$ is given by

$$
F=\left(\int_{0}^{\pi} f(\theta) d \rho_{i j}(\theta)\right),
$$

with $f(\theta)$ defined by (2) and (1). In particular, if $c_{1}=1$ and $c_{n}=0$ for $n>1$, then $f(\theta)=2 \cos \theta$ and (5) is the spectral resolution of $J$ (with the usual parameter $\lambda$ being given by $\lambda=2 \cos \theta$ ).

It should be noted that the $L^{2}$ assumption on the sequence $\left\{c_{n}\right\}$ in (1) does not imply the boundedness of the various matrices considered above, although of course, the existence, in the mean, of the integrals in (5) is assured. Moreover, all two factor products of the type $A^{2}$, $A A^{*}$, etc. surely exist and it can be verified that

Received August 26, 1958. This research was supported by the United States Air Force through the Air Force Office of Scientific Research of the Air Research and Development Command, under Contract No. AF 18 (603)-139. Reproduction in whole or in part is permitted for any purpose of the United States Government. 
where $A, T$ and $H$ are defined by (3); cf. [11], p. 517.

It is known (Toeplitz) that $T$ is bounded if and only if $f(\theta)$ is essentially bounded, so that $|f(\theta)| \leq$ const. almost everywhere on $[0, \pi]$; [3], p. 360. Moreover, if $M$ and $m$ denote the essential upper and lower bounds of $f$, then the spectrum of $T$ consists of the interval $[m, M]$ and, unless all $c_{n}=0$, is purely continuous (so that the point spectrum is empty); [3] and [4]. Furthermore, if $T$ is not bounded, but is still selfadjoint, then the spectrum of $T$ is again purely continuous and is again the (unbounded) interval $[m, M] ;[4]$, p. 878. (Actually the results of Hartman and Wintner mentioned above are not restricted to the case of real sequences $\left\{c_{n}\right\}$ as in the present paper.) For necessary and sufficient conditions in order that a Hankel matrix be bounded, see [9].

The matrix $A$ is bounded if and only if $g(\theta) \sim \sum_{1}^{\infty} c_{n} e^{i n \theta}$ is essentially bounded (Toeplitz; cf. [4], p. 880, [11], p. 517). Clearly, if $A$ is bounded, so also is $T$. In addition, if $T$ is bounded, then $f(\theta)$ of (2) is bounded and consequently $K$ is bounded (Toeplitz; cf. [2], p. 223, also [3], p. 365). In view of the easily verified relation

$$
\|H x\|^{2}=\|K x\|^{2}+\left(\sum_{1}^{\infty} c_{n} x_{n}\right)^{2}
$$

$H$ is bounded (or completely continuous) if and only if $K$ is bounded (or completely continuous). It is seen that if $A$ is bounded then all of the other matrices considered above are bounded.

In $\S 2$ there will be pointed out a few consequences of the relations (4) and (5) bearing on the nature of Fourier series and the spectra of Toeplitz matrices belonging to real valued, even functions defined by (2). In $\S \S 3$ and 4 , sufficient conditions guaranteeing that a Toeplitz matrix be absolutely continuous or unitarily equivalent to a certain function of $J$, will be obtained. Some applications to Hilbert matrices will be given in $\S 5$.

Condition (1) on the real sequence $\left\{c_{n}\right\}$ will be assumed throughout the paper.

2. Toeplitz matrices and Fourier series. First there will be proved:

(I) If the Hankel matrix $K$ is bounded, then necessarily $T$ is selfadjoint.

This is an obvious consequence of (4) if it is noted that $F$ of (5) is always self-adjoint. Incidentally, it is seen that in this case the domain of $T$ is identical with that of $F$.

By the essential range of $f(\theta)$ will be meant the (closed) set of values $\lambda$ for which $|f(\theta)-\lambda|<\varepsilon$ holds on a set of positive measure 
on $[0, \pi]$ for every $\varepsilon>0$. Then one has:

(II) If $K$ is bounded and satisfies $\|K\| \leq C$ (= const.), then any closed interval of length $2 C$ contained in $[m, M]$ contains at least one point of the essential range of $f(\theta)$.

This assertion also follows from (4). For, it is clear that the spectrum of $F$ is exactly the essential range of $f(\theta)$ (the situation being analogous to the problem of Toeplitz of determining the spectrum of a Laurent matrix; cf. [3], pp. 359-360 and the references cited there). Consequently, since relation (4) shows that $F$ is obtained as a perturbation of $T$ by the operator $-K$, the assertion now follows from the fact that the spectrum of $T$ is the entire interval $[m, M]$.

A theorem similar to (II) is

(III) If $K$ is completely continuous then the essential range of $f(\theta)$ is $[m, M]$.

The proof follows from the observation that, by (the generalization of) Weyl's theorem, the essential (cluster) spectra of $T$ and $F$ are identical. Since, by (I), $T$ is self-adjoint, its spectrum is $[m, M]$ ([4]) and it follows that the essential spectrum of $F$ is also $[m, M]$. But the spectrum of $F$ is always contained in $[m, M]$ and hence must be identical with this interval. This implies (III).

A curious corollary of (III) is the following assertion:

(IV) If $g(\theta) \sim \sum_{1}^{\infty} c_{n} \sin n \theta$ is continuous on $-\infty<\theta<\infty$ then the essential range of $h(\theta) \sim \sum_{1}^{\infty} c_{n} \cos n \theta$ is an interval (possibly unbounded).

The assertion follows from (III) if it is noted that the continuity of $g(\theta)$ implies the complete continuity of the operator $K$. Cf. [3], p. 365 . It is stated there that $H=\left(c_{i+j-1}\right)$ is completely continuous if either $g(\theta)$ or the function $\frac{1}{2} f(\theta) \sim \sum_{1}^{\infty} c_{n} \cos n \theta$ is continuous on $[0, \pi]$. The proof seems to indicate however that $K=\left(c_{i+j}\right)$ (or $H$ ) is completely continuous if either $g(\theta)$ or $f(\theta)$ is continuous on $[-\pi, \pi]$ (and hence on $-\infty<\theta<\infty$ ). See [2], p. 223. The continuity of $g(\theta)$ on $[0, \pi]$ implies its continuity on $[-\pi, \pi]$ but the corresponding assertion for $f(\theta)$ is false.

Another easy consequence of (4) is the following:

(V) If $T_{1}$ and $T_{2}$ are two bounded Toeplitz matrices with the representations

$$
\left(4_{m}\right) \quad T_{m}=F_{m}+K_{m}
$$


$\left(5_{m}\right) \quad F_{m}=\left(\int_{0}^{\pi} f_{m}(\theta) d \rho_{i j}(\theta)\right)$ and $f_{m}(\theta) \sim 2 \sum_{1}^{\infty} c_{m n} \cos n \theta$, and if $K_{1}$ and $K_{2}$ are completely continuous, then

$$
T_{1} T_{2}=\left(\int_{0}^{\pi} f_{1}(\theta) f_{2}(\theta) d \rho_{i j}(\theta)\right)+C,
$$

where $C$ is completely continuous.

A relation similar to (8) holds of course for products with more than two factors. The proof of (V) follows easily if it is noted that the product of a bounded operator and a completely continuous operator is completely continuous. In particular, it is seen from (8) that the essential spectrum of $T_{1} T_{2}$ is the essential range of $f_{1}(\theta) f_{2}(\theta)$. The situation is to be compared with that for Laurent matrices; cf. the remark following (II) above.

3. Absolute continuity. It follows from Theorem 13 of [11], p. 523, that if $A$ is bounded, then (6) implies that $T$ is absolutely continuous whenever 0 is not in the point spectrum of $H$. That is, this last condition is sufficient in order to guarantee that $\int_{Z} d E(\lambda)=0$, where

$$
T=\int \lambda d E(\lambda)
$$

is the spectral resolution of $T$ and $Z$ is any set of one-dimensional Lebesgue measure zero. However it is possible that $T$ is absolutely continuous even if 0 does belong to the point spectrum of $H$. In fact each $T_{N}$, belonging to the sequence $\left\{c_{n}\right\}$ with $c_{n}=c_{N} \neq 0(N>0)$ if $n= \pm N$ and $c_{n}=0$ otherwise, is absolutely continuous; cf. [11], pp. 519, 524. This result will be generalized in the following theorem:

(VI) Let the real sequence $\left\{c_{n}\right\}, n=0, \pm 1, \pm 2, \cdots$ satisfy (1) define the associated matrices $A, T$ and $F$ as in $\S 1$, and suppose that $A$ is bounded. Then $T$ is absolutely continuous whenever $F$ is absolutely continuous.

As remarked above, the boundedness of $A$ implies that of all other operators considered. It follows from the argument of [10] (cf. p. 1027, formula line (4)) when applied to (6) that

$$
H \int_{Z} d E(\lambda)=0
$$

where $Z$ denotes any set of one-dimensional Lebesgue measure zero and $H$ is defined by (3). (The square root $H^{1 / 2}$ appearing in [10] loc. cit. can clearly be taken to be any self-adjoint square root of the non-negative 
self-adjoint operator $H$. The $H$ appearing there corresponds to a positive multiple of the operator $H^{2}$ of the present paper.) Next let $y$ be any element in the range of $\int_{Z} d E(\lambda)$ so that

$$
y=\int_{Z} d E(\lambda) x, \quad \quad\|x\|<\infty .
$$

Since, by (10), $H y=0$, it follows from (7) that $K y=0$. Consequently, by (4), $T y=F y$. For $n=0,1,2, \cdots, T^{n} y=\int_{z} d E(\lambda) T^{n} x$ is also in the range of $\int_{Z} d E(\lambda)$, and it follows that $T^{n+1} y=F T^{n} y$. Hence

$$
T^{n} y=F^{n} y \quad\left(n=0,1,2, \cdots ; T^{0}=F^{0}=I\right),
$$

where $y$ is defined by (11). But (12) implies $E(\lambda) y=F(\lambda) y$, where

$$
F=\int \lambda d F(\lambda)
$$

is the spectral resolution of $F$, and hence $\int_{Z} d E(\lambda) y=\int_{Z} d F(\lambda) y$. But, whenever $F$ is absolutely continuous, $\int_{Z} d F(\lambda)=0$ and so, by (11), $\int_{z} d E(\lambda) x=0$ for all $x$. That is, $T$ is absolutely continuous and the proof of (VI) is now complete.

4. Unitary equivalence. It was shown in [11] that each $T_{N}$ (see the beginning of $\S 3$ above) is absolutely continuous and that moreover $T_{N}$ is unitarily equivalent to the corresponding $F=F_{N}$. This result will be considerably refined in the following theorem:

(VII) Let the real sequence $\left\{c_{n}\right\}$ satisfy (1) and the condition

$$
\left|c_{n}\right| \leq \text { const. } \alpha^{n}
$$$$
(n=1,2, \cdots)
$$

for some constant $\alpha, 0<\alpha<1$. Then the associated matrices $T$ and $F$ are unitarily equivalent; thus, there exists a unitary matrix $U$ such that

$$
T=U F U^{*} .
$$

The condition (14) easily assures $\sum\left|c_{n}\right|<\infty$ and hence the boundedness of $A$ and therefore (cf. $\S 1$ above) that of all other operators considered. If all $c_{n}=0$, then $T$ and $F$ are both the zero operator (matrix) and (15) is trivial. Suppose then that not all $c_{n}$ are 0 . It will first be shown that $F$ is absolutely continuous.

To this end, consider $f(z)=2 \sum_{1}^{\infty} c_{n} \cos n z$ for the complex variable 
$z=x+i y$. It is clear that $|\cos n z|=\frac{1}{2}\left|e^{i n z}+e^{-i n z}\right| \leq \frac{1}{2}\left(e^{n y}+e^{-n y}\right) \leq$ $e^{n|y|}$ and hence, by (14), $\left|c_{n} \cos n z\right| \leq$ const. $\left(\alpha e^{|y|}\right)^{n}$. Since $0<\alpha<1$, it follows that $\alpha e^{|y|}<1$ for $y$ sufficiently small and so $f(z) \not \equiv 0$ and is analytic in a strip containing the real axis. Consequently $d f(\theta) / d \theta$ can be zero at most a finite number of times on $0 \leq \theta \leq \pi$ and it follows that the (possibly many-valued) inverse function of $f(\theta)$ on $[0, \pi]$ is absolutely continuous (more precisely, that each of the finite number of branches of the inverse of $f(\theta)$ on $0 \leq \theta \leq \pi$ is absolutely continuous). Moreover, if $\lambda=2 \cos \theta$, the operator $F$ can be represented (cf. (5)) as $F=\int_{-2}^{2} h(\lambda) d E_{1}(\lambda)$ where $E_{1}(\lambda)$ is the resolution of the identity belonging to the matrix $J$. Since $h(\lambda)=f(\theta)$ via the substitution $\lambda=2 \cos \theta$ it is clear that $h(\lambda)$ has a (possibly many-valued) absolutely continuous inverse and it follows (cf. [11], pp. 521-522) that $F$ is absolutely continuous, as was to be shown. In fact, if one considers the spectral resolution of $F$ as given by (13), it is seen from a comparison with (5) that zero sets on the $\lambda$-interval $-2 \leq \lambda \leq 2$ correspond to zero on the $\theta$-interval $0 \leq \theta \leq \pi$ via the mapping $\lambda=2 \cos \theta$ and that $F$ is absolutely continuous if and only if the relation

$$
\{\theta ; f(\theta) \text { in } Z\} \text { is a zero set }
$$

holds whenever $Z$ is a zero set.

By (VI) it now follows that $T$ also is absolutely continuous. Moreover, since by (14),

$$
\sum_{i} \sum_{j} c_{i+j}^{2}=\sum_{n} n c_{n+1}^{2}<\infty,
$$

$K$ is completely continuous. In order to complete the proof it will be shown that

$$
\operatorname{tr}|K|<\infty,
$$

where $|K|$ denotes the non-negative square root of $K^{2}$. An application of a theorem of Rosenblum ([12], p. 998, will then yield the desired unitary equivalence relation (15). See also Kato [6].

There remains then to prove (17). Let $\left\{\phi_{n}\right\}, n=1,2, \cdots$, denote the complete orthonormal sequence of vectors for which the $n$-th component of $\phi_{n}$ is 1 and all others are 0 . Then

$$
\begin{aligned}
\operatorname{tr}|K| & =\sum\left(|K| \phi_{n}, \phi_{n}\right) \leq \sum\left\||K| \phi_{n}=\sum\right\| K \phi_{n} \| \\
& =\sum_{n}\left(\sum_{m} c_{n+m}^{2}\right)^{1 / 2} \leq \sum_{n} \sum_{m}\left|c_{n+m}\right| \leq \sum n\left|c_{n+1}\right|<\infty,
\end{aligned}
$$

the last inequality by (14). Thus (17) is proved and, as remarked earlier, the proof of (VII) is complete. 
The proof of (VII) makes clear the following assertion:

(VIII) Let the real sequence $\left\{c_{n}\right\}$ satisfy (1) and suppose that (16) holds for every zero set $Z$. In addition, suppose that

$$
\sum_{n} n\left|c_{n+1}\right|<\infty, \text { or even } \sum_{n}\left(\sum_{m} c_{n+m}^{2}\right)^{1 / 2}<\infty .
$$

Then (15) holds.

It is clear that (18) implies $\sum\left|c_{n}\right|<\infty$ and hence that $A$ is bounded (cf. $\S 1$ above). In addition (18) implies $\sum n c_{n+1}^{2}<\infty$ and hence the complete continuity of $K$; as shown before, (18) implies (17). Moreover, unless $T$ and $F$ are both 0 , it follows from (16) that $F$ (hence, by (VI), also $T$ ) is absolutely continuous. Relation (15) now follows from Rosenblum's theorem as before.

It was shown in [4], p. 878, that whenever $T$ is self-adjoint (not even necessarily bounded) it has no point spectrum. On the other hand, $F$ has a point spectrum whenever $f(\theta)$ has an interval of constancy, or more generally, whenever $f(\theta) \equiv$ const. holds on a set of positive measure. This situation can of course easily obtain for non-trivial $f(\theta)(f(\theta) \not \equiv$ const., i.e., since $\left.c_{0}=0, f(\theta) \not \equiv 0\right)$ possessing derivatives of arbitrarily high order (but, of course, for which $f(z)$ is not analytic). But if $f(\theta)$ is of class $C^{p}$, its Fourier coefficients are $O\left(n^{-p-2}\right)$ and so it is clear that the hypothesis (14) of (VII) guaranteeing unitary equivalence cannot be weakened to, say, $\left|c_{n}\right| \leq$ const. $n^{-m}(n=1,2, \cdots)$ for any positive constant $m$. Of course, as (VIII) implies, relation (14) is not necessary for (15).

5. Hilbert matrices. A case of special interest is afforded by the sequence $\left\{c_{n}\right\}$ defined by $c_{0}=0, c_{n}=n^{-1}$ if $n>0$ and $c_{-n}=c_{n}$. This sequence is of the type considered at the beginning of this paper and moreover $T=\left(|i-j|^{-1}\right), H=\left((i+j-1)^{-1}\right)$ and $S=A-A^{*}=\left((i-j)^{-1}\right)$, with the understanding of course that the $(i, i)$ elements of $T$ and $S$ are 0 . The matrices $S$ and $H$ are known to be bounded (Hilbert; cf., e.g., [2], pp. 212-213, 223). Moreover, the spectrum of $H$ is exactly the interval $[0, \pi]$ and, in fact, is purely continuous ([6]). The matrix $T$ is known to be unbounded ([2]), p. 214). Concerning $T$, there will be proved the following theorem:

(IX) The matrix $T=\left(|i-j|^{-1}\right)$ is a self-adjoint operator and is absolutely continuous; thus if $T=\int \lambda d E(\lambda)$ is the spectral resolution of $T$, then $\int_{Z} d E(\lambda)=0$ for every set $Z$ of one-dimensional Lebesgue measure zero.

That $Z$ is self-adjoint follows from an application of a theorem of 
Hartman and Wintner [4], p. 878, if it is noted that $\frac{1}{2} f(\theta)=-\log \left(2\left|\sin \frac{1}{2} \theta\right|\right)$ $\sim \sum_{1}^{\infty} n^{-1} \cos n \theta$ on $(-\pi, \pi)$ is half-bounded. Another proof of the assertion follows from (I) if it is noted that $H$, hence also $K$ (cf. (7)), is bounded, since the odd function $g(\theta)$ defined by $g(\theta)=\frac{1}{2}(\pi-\theta)$ $\sim \sum_{1}^{\infty} n^{-1} \sin n \theta$ on $(0, \pi)$ is bounded.

It is easy to verify that

$$
S T-T S=2 H^{2},
$$

a relation similar to (6). Moreover, since 0 is not in the point spectrum of $H$ (cf., e.g. [7], p. 699 and the reference there to [1]), Theorem 13 of [11] implies, at least formally, the absolute continuity of $T$. The trouble stems from the fact that boundedness restrictions were imposed in [10] and [11] and that, although $S$ and $H$ in (19) are bounded, $T$ is not. As a consequence, equation (19), although a valid matrix equation, conceivably cannot be regarded as an operator equation in Hilbert space. More precisely, it is not clear that whenever $x$ is in the domain of $T$, $D_{T}$, then (19) holds, so that

$$
S T x-T S x=2 H^{2} x,
$$

with the understanding that $S T x$ and $T S x$ of (20) should mean $S(T x)$ and $T(S x)$ respectively. (For operator equations the associative law is of course essentially a matter of definition.) It will be shown below that in fact (20) does hold as an operator equation valid at least for all $x$ in $D_{T}$. Once this has been established, it is easy to carry out the same reasoning as in [10], cf. pp. 1027-1028 (where the boundedness of all operators was supposed) and to obtain the equation (10) above, corresponding to formula line (4) in [10]. The absolute continuity of $T$ then follows (ef. Theorem 13 of [11]) from the fact that 0 is not in the point spectrum of $H$.

In order to complete the proof there remains to be shown that if $x$ is in $D_{T}$ then (20) holds. To this end, it will be shown that if $x$ is in Hilbert space, that is if $\|x\|<\infty$, then each of the series

$$
\sum_{m} \sum_{n} s_{i m} t_{m n} x_{n} \text { and } \sum_{m} \sum_{n} t_{i m} s_{m n} x_{n}
$$

is absolutely convergent for $i=1,2, \cdots$, where, for convenience, $T=$ $\left(t_{i j}\right)$ and $S=\left(s_{i j}\right)$. Grant, for the moment, that this has been shown. Then, from the absolute convergence of the first series of (21), it follows that in the iterated series the orders of summation may be interchanged, and hence that, for $x$ in Hilbert space, the corresponding components of the vectors $(S T) x$ and $S(T x)$ are identical. Now, if it is assumed in addition that $x$ is in $D_{T}$, then the vector $S(T x)$ is in Hilbert space, since $S$ is bounded. Consequently $(S T) x$ is in Hilbert space and, since $H^{2}$ is 
bounded, it follows from the (matrix) equation (19) that (TS) $x$ is in Hilbert space. The absolute convergence of the second series of (21) then implies that $(T S) x=T(S x)$, so that $T(S x)$ is in Hilbert space (that is, essentially, that $S x$ is in $D_{T}$ ). Moreover, it is now seen that (19) implies the validity of (20) as an operator equation valid at least for all vectors in $D_{r}$.

Thus, in order to complete the proof of (IV) there now remains to be shown that the series of (21) are absolutely convergent whenever $\|x\|<\infty$. Consider the series $S_{i}=\sum_{m} \sum_{n}\left|s_{i m} t_{m n} x_{n}\right|$. Since $t_{i}, \geq 0$ for all $i, j$ and $s_{i j} \geq 0$ or $s_{i j}<0$ according as $i \geq j$ or $i<j$, it is clear that

$$
S_{i}=-\sum_{n=1}^{\infty}\left(\sum_{m=1}^{i} s_{i m} t_{m n}\left|x_{n}\right|\right)+\sum_{n=1}^{\infty}\left(\sum_{m=i+1}^{\infty} s_{i m} t_{m n}\left|x_{n}\right|\right) .
$$

But the inside series of the first double series on the right of equation (22) is finite and, consequently, the orders of summation may be interchanged to obtain $-\sum_{m=1}^{i} s_{i m}\left(\sum_{n=1}^{\infty} t_{m n}\left|x_{n}\right|\right)$. Since $x$ and the rows of $T$ are in Hilbert space, the inside summation of this last series is always convergent by the Schwarz inequality. Hence the first series of (21) is absolutely convergent if and only if the series

$$
\sum_{n=1}^{\infty}\left(\sum_{m=1}^{\infty} s_{i m} t_{m n}\right)\left|x_{n}\right|
$$

obtained through modification of $S_{i}$ in (22) by changing the sign of the first series, is convergent. Now the inside summation of (23) is the $(i, n)$ element of $S T=D=\left(d_{i n}\right)$. Since $S$ is bounded and the columns of $T$ are in Hilbert space, the columns of $D$ are in Hilbert space, that is $\sum_{i} d_{i n}^{2}<\infty$. But the matrix equation (19) can be written as $D+D^{*}=$ $2 H^{2}$; hence, since $H^{2}$ is bounded, the columns of $D^{*}$ and therefore the rows of $D$, are also in Hilbert space. Hence $\Sigma_{n} d_{i n}^{2}<\infty$ and so (23) is convergent by the Schwarz inequality. It has now been proved that the first series of (21) is absolutely convergent (for $i=1,2, \cdots$ ). Using the fact that $\left|s_{i j}\right|=t_{i j}$ it is seen that the absolute convergence of the first series of (21), that is, the convergence of $\sum_{m} \sum_{n} t_{i m} t_{m n}\left|x_{n}\right|$, whenever $\|x\|<\infty$, implies the absolute convergence of the second series of (21). Thus both series are convergent for all $x$ in Hilbert space and (IX) follows as indicated above.

\section{REFERENCES}

1. T. Carleman, Sur les équations intégrales singulières a noyau réel el symétrique, Uppsala, 1923.

2. G. H. Hardy, J. E. Littlewood, and G. Polya, Inequalities, Cambridge, 1934.

3. P. Hartman and A. Wintner, On the spectra of Toeplitz's matrices, Amer. J. Math., 72 (1950), 359-366.

4. P. Hartman and A. Wintner, The spectra of Toeplitz's matrices, Amer. J. Math., 
(1954), pp. 867-882.

5. D. Hilbert, Grundzüge einer allgemeinen Theorie der linearen Integralgleichuugen, Leipzig, 1912.

6. T. Kato, Perturbation of continuous spectra by trace class operators, Proc. Japan Academy, 33 (1957), 260-264.

7. W. Magnus, On the spectrum of Hilbert's matrix, Amer. J. Math., 72 (1950), 699-704.

8. Mathematical Monographs, Northwestern University, vol. 1, 1941.

9. Z. Nehari, On bounded bilinear forms, Annals of Math., 65 (1957), 153-162.

10. C. R. Putnam On commutators and Jacobi matrices Proc. Amer. Math. Soc., 7 (1956), 1026-1030.

11. - Commutators and absolutely continuous operators, Trans. Amer. Math. Soc., 87 (1958), 513-525.

12. M. Rosenblum, Perburbations of the continuous spectrum and unitary equivalence, Pacific J. Math., 7 (1957), 997-1010.

13. O. Toeplitz, Zur Theoric der quadratischen und bilinearen Formen von unendlichvielen Veränderlichen, Göttinger Nachrichten (1910), 489-506.

PURDUE UNIVERSITY 


\section{PACIFIC JOURNAL OF MATHEMATICS}

\section{EDITORS}

\section{David Gilbarg}

Stanford University Stanford, California

\section{R. A. Beaumont}

University of Washington

Seattle 5, Washington

\section{A. L. Whiteman}

University of Southern California Los Angeles 7, California

L. J. Paige

University of California

Los Angeles 24, California

\section{ASSOCIATE EDITORS}

E. F. BECKENBACH
C. E. BURGESS
E. HEWITT
A. HORN

A. HORN

\author{
V. GANAPATHY IYER \\ R. D. JAMES \\ M. S. KNEBELMAN \\ L. NACHBIN
}

I. NIVEN

T. G. OSTROM

H. L. ROYDEN

M. M. SCHIFFER
E. G. STRAUS

G. SZEKERES

F. WOLF

K. YOSIDA

\section{SUPPORTING INSTITUTIONS}

\author{
UNIVERSITY OF BRITISH COLUMBIA \\ CALIFORNIA INSTITUTE OF TECHNOLOGY \\ UNIVERSITY OF CALIFORNIA \\ MONTANA STATE UNIVERSITY \\ UNIVERSITY OF NEVADA \\ OREGON STATE COLLEGE \\ UNIVERSITY OF OREGON \\ OSAKA UNIVERSITY \\ UNIVERSITY OF SOUTHERN CALIFORNIA
}

\author{
STANFORD UNIVERSITY \\ UNIVERSITY OF TOKYO \\ UNIVERSITY OF UTAH \\ WASHINGTON STATE COLLEGE \\ UNIVERSITY OF WASHINGTON \\ * * * * \\ AMERICAN MATHEMATICAL SOCIETY \\ CALIFORNIA RESEARCH CORPORATION \\ HUGHES AIRCRAFT COMPANY \\ SPACE TECHNOLOGY LABORATORIES
}

Mathematical papers intended for publication in the Pacific Journal of Mathematics should be typewritten (double spaced), and the author should keep a complete copy. Manuscripts may be sent to any one of the four editors. All other communications to the editors should be addressed to the managing editor, L. J. Paige at the University of California, Los Angeles 24, California.

50 reprints per author of each article are furnished free of charge; additional copies may be obtained at cost in multiples of 50 .

The Pacific Journal of Mathematics is published quarterly, in March, June, September, and December. The price per volume (4 numbers) is $\$ 12.00$; single issues, $\$ 3.50$. Back numbers are available. Special price to individual faculty members of supporting institutions and to individual members of the American Mathematical Society: $\$ 4.00$ per volume; single issues, $\$ 1.25$.

Subscriptions, orders for back numbers, and changes of address should be sent to Pacific Journal of Mathematics, 2120 Oxford Street, Berkeley 4, California.

Printed at Kokusai Bunken Insatsusha (International Academic Printing Co., Ltd.), No. 6, 2-chome, Fujimi-cho, Chiyoda-ku, Tokyo, Japan.

PUBLISHED BY PACIFIC IOURNAL OF MATHEMATICS, A NON-PROFIT CORPORATION

The Supporting Institutions listed above contribute to the cost of publication of this Journal, but they are not owners or publishers and have no responsibility for its content or policies. 


\section{Pacific Journal of Mathematics}

\section{Vol. 9, No. $3 \quad$ July, 1959}

Errett Albert Bishop, A minimal boundary for function algebras . . . . . . . . . . . . 629

John W. Brace, The topology of almost uniform convergence . . . . . . . . . . . . 643

Cecil Edmund Burgess, Chainable continua and indecomposability .......... 653

L. Carlitz, Multiplication formulas for products of Bernoulli and Euler

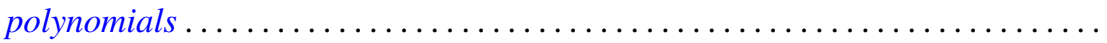

Eckford Cohen, A class of residue systems (mod $r$ ) and related arithmetical

functions. II. Higher dimensional analogues ....................

Shaul Foguel, Boolean algebras of projections of finite multiplicity . . . . . . . . . .

Richard Robinson Goldberg, Averages of Fourier coefficients .................

Seymour Goldberg, Ranges and inverses of perturbed linear operators .

Philip Hartman, On functions representable as a difference of convex functions ....

Milton Vernon Johns, Jr. and Ronald Pyke, On conditional expectation and

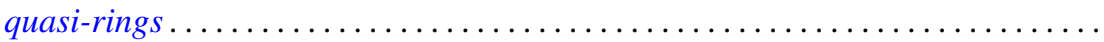

Robert Jacob Koch, Arcs in partially ordered spaces ....................

Gregers Louis Krabbe, A space of multipliers of type $L^{p}(-\infty, \infty) \ldots \ldots \ldots \ldots$

John W. Lamperti and Patrick Colonel Suppes, Chains of infinite order and their

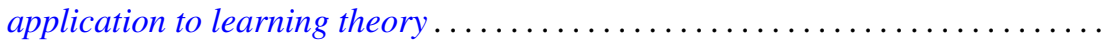

Edith Hirsch Luchins, On radicals and continuity of homomorphisms into Banach

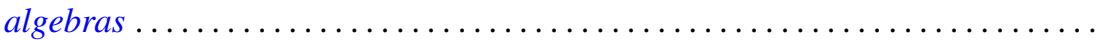

T. M. MacRobert, Multiplication formulae for the E-functions functions of their parameters.

Michael Bahir Maschler, Classes of minimal and representative domains and their kernel functions.

William Schumacher Massey, On the imbeddability of the real projective spaces in Euclidean space.

Thomas Wilson Mullikin, Semi-groups of class $\left(C_{0}\right)$ in $L_{p}$ determined by parabolic differential equations

Steven Orey, Recurrent Markov chains

Ernest Tilden Parker, On quadruply transitive groups ........ . .

Calvin R. Putnam, On Toeplitz matrices, absolute continuity, and unitary

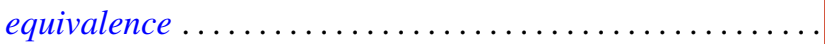

Helmut Heinrich Schaefer, On nonlinear positive operators.

Robert Seall and Marion Wetzel, Some connections between continued fractions and convex sets

Robert Steinberg, Variations on a theme of Chevalley

Olga Taussky and Hans Zassenhaus, On the similarity transformation between a

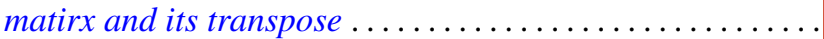

Emery Thomas, The suspension of the generalized Pontrjagin cohomology

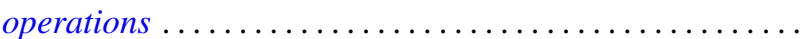

Joseph L. Ullman, On Tchebycheff polynomials ..................... 913

Richard Steven Varga, Orderings of the successive overrelaxation scheme ........ 925

Orlando Eugenio Villamayor, Sr., On weak dimension of algebras . 